\title{
Role of benzoic and salicylic acids in the immunization of oil palm seedlings-challenged by Ganoderma boninense
}

\begin{abstract}
Basal stem rot (BSR) infection of oil palm, caused by Ganoderma boninense, is one of the key constraining components in palm oil production causing considerable economic losses around the world. Although it was reported a century before, till date no effective controller has been identified, the alteration of lignin content in oil palm can control the BSR is one of the hypothesis. Since the degradation of lignin is the rate limiting step in the infection process of BSR disease. Two naturally occurring phenolic compounds such as benzoic acid and salicylic acid are known to play a key role in the plant defence as well as in the lignin synthesis. The goal of this research is to evaluate the effect of these two naturally occurring phenolic compounds against $\mathrm{G}$. boninense. In this study, oil palm seedlings were immunized with various concentrations (1-15 $\mathrm{mM}$ ) of benzoic acid and salicylic acid, prior to G. boninense inoculation. After challenging the oil palm seedlings with G. boninense, BSR progression along with the changes in defence enzymes (Phenylalanine ammonia lyase, Peroxidase, and Polyphenol oxidase), and the total lignin contents were examined and evaluated. The exogenous application of the phenolic compounds have not only suppressed the BSR infection but also promoted the plant growth significantly $(\mathrm{P} \leq 0.01)$. The disease suppression was due to the increased production of defence enzymes particularly polyphenol oxidase which had increased lignin content in the seedlings. An absolute disease reduction was registered in seedlings treated 10 and $15 \mathrm{mM}$ benzoic acid with a significant $(\mathrm{P} \leq 0.01)$ increment in the production of lignin along with the defence enzymes. Whereas the salicylic acid exhibited an ambivalent behavior, at $1 \mathrm{mM}$ salicylic acid induced the disease by $60 \%$ however as the concentration had increased to $5 \mathrm{mM}$ suppression in the BSR was observed. The efficiency of phenolic compounds as an agent of disease reduction is found to be concentration dependent. The outcome of this study has found that benzoic acid at $15 \mathrm{mM}$ concentration is a superior controller for the BSR disease suppression in oil palm. This study would pave the way towards a new management strategy to replace the chemical controls by naturally occurring phenolic compounds to control BSR effectively in oil palm ranches.
\end{abstract}

Keyword: Ganoderma boninense; Benzoic acid; Salicylic acid; Defence enzyme; Polyphenol oxidase; Lignin 